\title{
Diffraction of aperiodic crystals: the state of the art
}

\author{
Uwe Grimm \\ School of Mathematics and Statistics, The Open University, Walton Hall, Milton Keynes MK7 6AA, United Kingdom \\ uwe.grimm@open.ac.uk
}

Since the discovery of quasicrystals in the early 1980s, the diffraction of aperiodically ordered structures has been a fruitful area of research in mathematical crystallography and, increasingly, in mathematics; see [1] for general background. The characterisation of the diffraction of a structure is closely linked to the question of how to define a crystal, and indeed how to define the concept of order in general [2]. For the purpose of this talk, I shall adopt a rather general perspective, in the sense that I shall consider mainly purepoint diffractive systems, but also talk about systems with singular continuous or absolutely continuous diffraction. While some of these systems may not be realised in nature, they are increasingly of interest as metamaterials, with the intention to obtain materials with purpose-made properties.

In this talk, I shall present an overview on the current state of knowledge on the diffraction of aperiodic structures [3,4]. Because the mathematics behind some of the results is non-trivial, I shall try to motivate and explain the results by means of explicit example systems, using some rather familiar as well as some less familiar examples to demonstrate what can happen.

This includes, on the one hand, examples of cut-and-project sets, which are aperiodic structures obtained by projection of part of a higher-dimensional lattice. For such systems, which include familiar examples such as the one-dimensional Fibonacci system or planar Penrose tilings, the theory of diffraction is rather well understood. On the other hand, there are self-similar structures that are obtained by an (iterative) inflation procedure, for which diffraction is, in general, much less well understood [5]. For the latter, recent work on using a renormalisation-type approach, which exploits the self-similarity, provides some new insight [6]. Particularly interesting examples are systems which possess a description as a projection set as well as an inflation symmetry, and I shall finish with the discussion of a class of examples of such structures, which is based on a two-dimensional Fibonacci system [7].

[1] Baake, M., Grimm, U. (2013). Aperiodic Order. Vol 1: A Mathematical Invitation. Cambridge: Cambridge University Press.

[2] Grimm, U. (2015). Aperiodic crystals and beyond. Acta Crystallogr. B 71, 258-274.

[3] Baake, M., Grimm, U. (2011). Kinematic diffraction from a mathematical viewpoint. Z. Kristallogr. 226, 711-725.

[4] Baake, M., Grimm, U. (2012). Mathematical diffraction of aperiodic structures. Chem. Soc. Rev. 41, 6821-6843.

[5] Baake, M., Grimm, U. (2020). Inflation versus projection sets in aperiodic systems: the role of the window in averaging and diffraction. Acta Crystallogr. A 76, 559-570.

[6] Baake, M., Frank, N. P., Grimm, U., Robinson, E.A. (2019). Geometric properties of a binary non-Pisot inflation and absence of absolutely continuous diffraction, Studia Math. 247, 109-154.

[7] Baake, M., Frank, N. P., Grimm, U. (2021). Three variations on a theme by Fibonacci. Stoch. Dyn. 21, 2410001.

Keywords: aperiodic order; quasicrystals; diffraction; higher-dimensional crystallography; mathematical crystallography

The author gratefully acknowledges support by the Engineering and Physical Sciences Research Council (EPSRC), who funded this work under grant reference EP/S010335/1. 